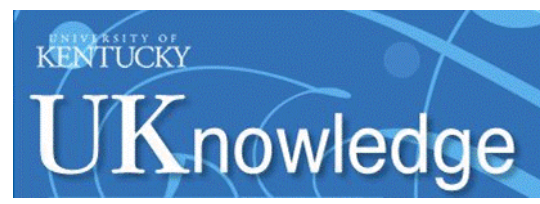

University of Kentucky

UKnowledge

8-14-2013

\title{
Long-term Spectral Evolution of Tidal Disruption Candidates Selected by Strong Coronal Lines
}

\author{
Chen-Wei Yang \\ Chinese Academy of Sciences, China \\ Ting-Gui Wang \\ Chinese Academy of Sciences, China \\ Gary J. Ferland \\ University of Kentucky, gary@uky.edu \\ Weimin Yuan \\ Chinese Academy of Sciences, China \\ Hong-Yan Zhou \\ Chinese Academy of Sciences, China
}

See next page for additional authors

Follow this and additional works at: https://uknowledge.uky.edu/physastron_facpub

Part of the Astrophysics and Astronomy Commons, and the Physics Commons

Right click to open a feedback form in a new tab to let us know how this document benefits you.

\section{Repository Citation}

Yang, Chen-Wei; Wang, Ting-Gui; Ferland, Gary J.; Yuan, Weimin; Zhou, Hong-Yan; and Jiang, Peng, "Longterm Spectral Evolution of Tidal Disruption Candidates Selected by Strong Coronal Lines" (2013). Physics and Astronomy Faculty Publications. 59.

https://uknowledge.uky.edu/physastron_facpub/59

This Article is brought to you for free and open access by the Physics and Astronomy at UKnowledge. It has been accepted for inclusion in Physics and Astronomy Faculty Publications by an authorized administrator of UKnowledge. For more information, please contact UKnowledge@lsv.uky.edu. 
Long-term Spectral Evolution of Tidal Disruption Candidates Selected by Strong Coronal Lines

Digital Object Identifier (DOI)

https://doi.org/10.1088/0004-637X/774/1/46

Notes/Citation Information

Published in The Astrophysical Journal, v. 774, no. 1, 46, p. 1-13.

(C) 2013. The American Astronomical Society. All rights reserved. Printed in the U.S.A.

The copyright holder has granted permission for posting the article here.

Authors

Chen-Wei Yang, Ting-Gui Wang, Gary J. Ferland, Weimin Yuan, Hong-Yan Zhou, and Peng Jiang 


\title{
LONG-TERM SPECTRAL EVOLUTION OF TIDAL DISRUPTION CANDIDATES SELECTED BY STRONG CORONAL LINES
}

\author{
Chen-Wei Yang ${ }^{1}$, Ting-Gui Wang ${ }^{1}$, Gary Ferland ${ }^{2}$, Weimin Yuan ${ }^{3}$, Hong-Yan Zhou ${ }^{1,4}$, and Peng Jiang ${ }^{1}$ \\ ${ }^{1}$ Key Laboratory for Research in Galaxies and Cosmology, The University of Sciences and Technology of China, \\ Chinese Academy of Sciences, Hefei, Anhui 230026, China; twang@ ustc.edu.cn \\ ${ }^{2}$ Department of Physics, University of Kentucky, Lexington, KY 40506, USA \\ ${ }^{3}$ National Astronomical Observatory, Chinese Academy of Sciences, 20A Datun Road, Beijing, China \\ ${ }^{4}$ Polar Research Institute of China, 451 Jinqiao Road, Pudong, Shanghai 200136, China \\ Received 2013 March 29; accepted 2013 July 9; published 2013 August 14
}

\begin{abstract}
We present results of follow-up optical spectroscopic Multi-Mirror Telescope (MMT) observations of seven rare, extreme coronal line-emitting galaxies reported by Wang et al. Large variations in coronal lines are found in four objects, making them strong candidates for tidal disruption events (TDEs). For the four TDE candidates, all the coronal lines with ionization states higher than [Fe VII] disappear within 5-9 yr. The [Fe VII] line faded by a factor of about five in one object $(\mathrm{J} 0952+2143)$ within $4 \mathrm{yr}$, whereas the line emerged in another two objects that previously did not show the line. A strong increment in the [O III] flux is observed, shifting the line ratios toward the loci of active galactic nuclei on the BPT diagram. Surprisingly, we detect a non-canonical [O III] $\lambda 5007 /[\mathrm{O} \mathrm{III]} \lambda 4959 \mathrm{ratio}$ of $\simeq 2$ in two objects, indicating a large column density of $\mathrm{O}^{2+}$ and thus probably optically thick gas. This result also requires a very large ionization parameter and a relatively soft ionizing spectral energy distribution (e.g., a blackbody with $T<5 \times 10^{4} \mathrm{~K}$ ). Our observations can be explained as the echoing of a strong ultraviolet to soft X-ray flare caused by TDEs on molecular clouds in the inner parsecs of the galactic nuclei. Reanalyzing the Sloan Digital Sky Survey spectra reveals double-peaked or strongly blue-shouldered broad lines in three of the objects, which disappeared in the MMT spectra of two objects and faded by a factor of 10 in $8 \mathrm{yr}$ in the remaining object with a decrease in both the line width and centroid offset. We interpret these broad lines as arising from decelerating biconical outflows. Our results demonstrate that the signatures of echoing can persist for as long as $10 \mathrm{yr}$ and can be used to probe the gas environment in quiescent galactic nuclei.
\end{abstract}

Key words: black hole physics - galaxies: nuclei - line: formation

Online-only material: color figures

\section{INTRODUCTION}

Stellar and gaseous kinematics suggest that most galaxies harbor central supermassive black holes (SMBHs) and that the SMBH masses are well correlated with the galactic stellar masses or velocity dispersions of the galactic bulges (e.g., Kormendy \& Richstone 1995; Magorrian et al. 1998; Ferrarese \& Merritt 2000; Gebhardt et al. 2000). When a star in the galactic nucleus accidentally passes within the tidal disruption radius of the massive black hole $\left(r_{p}<R_{T} \simeq R_{*}\left(M_{\mathrm{BH}} / M_{*}\right)^{1 / 3}\right.$; Hills 1975), it is torn apart by the tidal force of the black hole. About half of the stellar debris is ejected and the rest falls back toward the black hole, causing a bright flare lasting for a few months to years (Rees 1988; Ayal et al. 2000). For a black hole of mass between $10^{7} M_{\odot}$ and $10^{8} M_{\odot}$ and a solar-type star, the peak luminosity will be sub-Eddington $\left(L_{\mathrm{Edd}}=1.3 \times 10^{38}\left(M_{\mathrm{BH}} / M_{\odot}\right) \mathrm{erg} \mathrm{s}^{-1}\right)$. For a less massive black hole $\left(M_{\mathrm{BH}}<10^{7} M_{\odot}\right)$, the rate of fallback initially exceeds the Eddington accretion rate, likely launching strong outflows. The spectra of the super-Eddington accretion tori and outflows are still uncertain, but when the fallback rate is below the Eddington rate, the gas is accreted onto the black hole via a thin disk and its radiation peaks in the UV to soft X-ray bands.

Besides UV and soft X-ray continuum flares, variable broad and narrow emission lines may be also seen in the spectra. Part of the UV and X-ray light may be reprocessed by the outflows or unbound debris, giving rise to the broad emission lines (Bogdanović et al. 2004; Strubbe \& Quataert 2009; Wang et al. 2011, hereafter W11; Gezari et al. 2012). The same UV and
$\mathrm{X}$-ray sources may also ionize the cold circumnuclear interstellar medium (ISM), producing high-ionization narrow lines (Ulmer 1999; Komossa et al. 2008; Wang et al. 2011, 2012). Broad lines are expected to follow the continuum variations closely because they are produced in a region very close to the continuum source. Narrow lines should also be variable, but on a longer timescale due to the response of ISM material further away to the continuum flare (light echoing). In particular, the high-ionization narrow lines that vary on timescales of years are unique features of the light echo of tidal disruption events (TDEs). The high-ionization narrow lines distinguish a galaxy from an active galactic nucleus (AGN), which possesses a narrow line region (NLR) of a size ranging from $10^{2}$ to $10^{3} \mathrm{pc}$. Because gas at large radii will have little response to the continuum flare on timescales of months to years, we may expect to observe only the spectrum from the very inner region of the NLR, which is likely dominated by high-ionization lines, while low-ionization lines from the rest of the NLR are weak.

Wang et al. (2012, hereafter W12) carried out a systematic search for extreme coronal line emitters (ECLEs) in the spectroscopic samples of galaxies and low-redshift quasars from the Sloan Digital Sky Survey (SDSS) Data Release 7, following the initial results of detecting variable narrow lines in two ECLEs (Komossa et al. 2008, 2009; W11). W12 identified a sample of seven galaxies that showed extremely strong coronal lines from [Fe X] up to [Fe XIV]. Traditional narrow-line diagnostics suggested that the galaxies were non-active; half of the sample showed broad emission lines with complex profiles. Furthermore, the sample was split into low- and high-ionization 
Table 1

Basic Data of Extreme Coronal Line Emitters ${ }^{\mathrm{a}}$

\begin{tabular}{|c|c|c|c|c|c|c|c|c|c|c|}
\hline \multirow[t]{2}{*}{ No. } & \multirow[t]{2}{*}{ Name } & \multirow[t]{2}{*}{$z$} & \multirow[t]{2}{*}{$M_{i, \mathrm{tot}}^{\mathrm{b}}$} & \multirow[t]{2}{*}{ Obs Interval $^{\mathrm{c}}$} & \multirow[t]{2}{*}{ Broad Line } & \multicolumn{2}{|c|}{ [Fe VII] } & \multirow[t]{2}{*}{ [O III] } & \multicolumn{2}{|c|}{$\mathrm{NSC}^{\mathrm{d}}$} \\
\hline & & & & & & SDSS & MMT & & SDSS & MMT \\
\hline 1 & SDSS J074820.66+471214.6 & 0.0615 & -19.75 & 2866 & Fading & No & No & Increase & Yes & No \\
\hline 2 & SDSS J095209.56+214313.3 & 0.0789 & -20.41 & 2187 & Fading & Yes & Yes & Increase & No & No \\
\hline 3 & SDSS J134244.42+053056.1 & 0.0366 & -18.91 & 3548 & Absence & No & Yes & Increase & No & No \\
\hline 4 & SDSS J135001.49+291609.7 & 0.0777 & -19.76 & 2073 & Fading & No & Yes & Increase & No & No \\
\hline 5 & SDSS J093801.64+135317.0 & 0.1006 & -21.29 & 1834 & & & & & & \\
\hline 7 & SDSS J124134.26+442639.2 & 0.0419 & -19.95 & 2859 & & & & & & \\
\hline
\end{tabular}

Notes.

${ }^{a}$ First four objects show significant variations in the continuum and emission lines between two observations; the last three do not. We simply list the variations of the first four objects.

${ }^{\mathrm{b}} M_{i, \text { tot }}$ is estimated from SDSS photometry.

${ }^{c}$ Days between the SDSS and MMT observations.

d Non-stellar continuum.

subclasses according to the detection or non-detection of [Fe VII]. W12 showed that these lines are formed in photoionized gas, and argued that ECLEs are most likely the light echoes of a tidal disruption flare based on energetics considerations. Most of these galaxies are intermediate-luminosity disk galaxies with small bulges. If all these sources turn out to be TDE, then the rate of such TDEs is expected around a few times $10^{-5}$ per galaxy per year for galaxies with the absolute magnitudes in the range $-21.3<M_{i}<-18.9$.

However, there are some further questions left from previous studies. First, optical follow-up was carried out for only two of the seven objects. It remains to be verified whether the rest of the sample also shows the same variation trends. Second, the long-term evolution of the emission lines is not known. On what timescales do coronal lines disappear? Are there any other special features in the later stage of evolution that can be used to identify such events on long timescales? With these questions, we initiated spectroscopic follow-up observations with the Multi-Mirror Telescope (MMT) to characterize the spectral signatures at late phases of evolution. In this paper, we present an analysis of the follow-up MMT spectroscopic observations. The paper is organized as follows. We present the observations and data reduction in Section 2 and results on the spectral evolution in Section 3. We discuss the implication of these results in Section 4. Throughout the paper, we adopt a $\Lambda$-CDM cosmology with $H_{0}=71 \mathrm{~km} \mathrm{~s}^{-1} \mathrm{Mpc}^{-1}, \Omega_{M}=0.28$, and $\Omega_{\Lambda}=0.72$.

\section{OBSERVATIONS AND DATA REDUCTION}

We observed all seven targets with the Blue Channel Spectrograph mounted on the MMT on 2011 December 26. The observation log is shown in Table 1. During the observations, the typical seeing was 0.8 and we adopted a long slit with $1^{\prime \prime}$ width. The slit was centered on the galactic nuclei and orientated at the parallactic angle. We took exposures of all seven targets for $900 \mathrm{~s}$ with the $500 \mathrm{gpm}$ grating, centered at $6000 \AA$. This setting results in a spectral resolution $R=\lambda / \Delta \lambda=1430$ and wavelength coverage from $4430 \AA$ to $7560 \AA$. Unfortunately, the slit was not properly placed on the center of the galaxy for SDSS $\mathrm{J} 1241+4426$, thus we ignore the red part of spectrum for this object for all future analyses. We also observed the blue part of the spectrum for five objects, including SDSS J0748+4712, SDSS J0938+1353, SDSS J0952+2143, SDSS J1055+5637, and SDSS $\mathrm{J} 1241+4426$ using the 800 gpm grating centered at $4100 \AA$. The exposure time was $900 \mathrm{~s}$ for each target. A spectral resolution of 1730 and a wavelength coverage of $3100 \AA$ to $5100 \AA$ was obtained for the blue-side spectra. Three and one KPNO standard stars were observed using the same settings as the $500 \mathrm{gpm}$ and $800 \mathrm{gpm}$ setups during the observations for flux calibration, and seven and five $\mathrm{He}-\mathrm{Ne}-\mathrm{Ar}$ lamp spectra with the $500 \mathrm{gpm}$ and the 800 gpm grism sets were taken for the wavelength calibration. The raw two-dimensional (2D) data reduction and spectral extraction were accomplished using standard routines in IRAF. To extract the nuclear spectra, we used the APALL $\operatorname{task}^{5}$ and choose an aperture of $3^{\prime \prime}$. We also extracted the spectra with an aperture of $1^{\prime \prime}$; all high-ionization lines including the coronal lines, [O III], and He II $\lambda 4686$ are almost the same, so they all must come from the central $1^{\prime \prime}$ region. We carried out wavelength and flux calibration using the spectra of an $\mathrm{He}-\mathrm{Ne}-\mathrm{Ar}$ lamp and those of the standard stars, respectively. When applying different standard stars observed with the $500 \mathrm{gpm}$ set up for flux calibration, the spectra of the same object were consistent with each other to within 5\%. Thus, we took the medians as our final results. After the flux calibration, the blue and red spectra of the same object obtained with the two gratings were consistent with each other to within $8 \%$ in the overlapping region, indicating a small uncertainty in the flux calibration. Then, we rescaled the blue part of the spectrum to match the red part, and we then combined the two spectra. We estimated the spectral resolution by measuring the width of emission lines in the spectrum of the $\mathrm{He}-\mathrm{Ne}-\mathrm{Ar}$ lamp. For the $500 \mathrm{gpm}$ grating and the $800 \mathrm{gpm}$ grating, typical values are $90 \mathrm{~km} \mathrm{~s}^{-1}$ and $69 \mathrm{~km} \mathrm{~s}^{-1}$, respectively.

\section{RESULTS}

\subsection{Comparison between SDSS and MMT Spectra}

The MMT spectra are shown in Figure 1. For comparison, we also overlay the SDSS spectra taken 5-9 yr ago. Note that the SDSS spectra were obtained using a larger aperture (a circle 3." 0 in diameter) than the MMT spectra (a 1".0 $\times 3$.". 0 region), so the SDSS data contain more light from the host galaxy than our MMT spectra. As such, the low-ionization narrow lines from the extended star forming regions may be significantly smaller in the MMT spectra, along with the starlight. We do not expect,

\footnotetext{
5 APALL is a multi-step task that defines and extracts data from 2D CCD images in IRAF.
} 


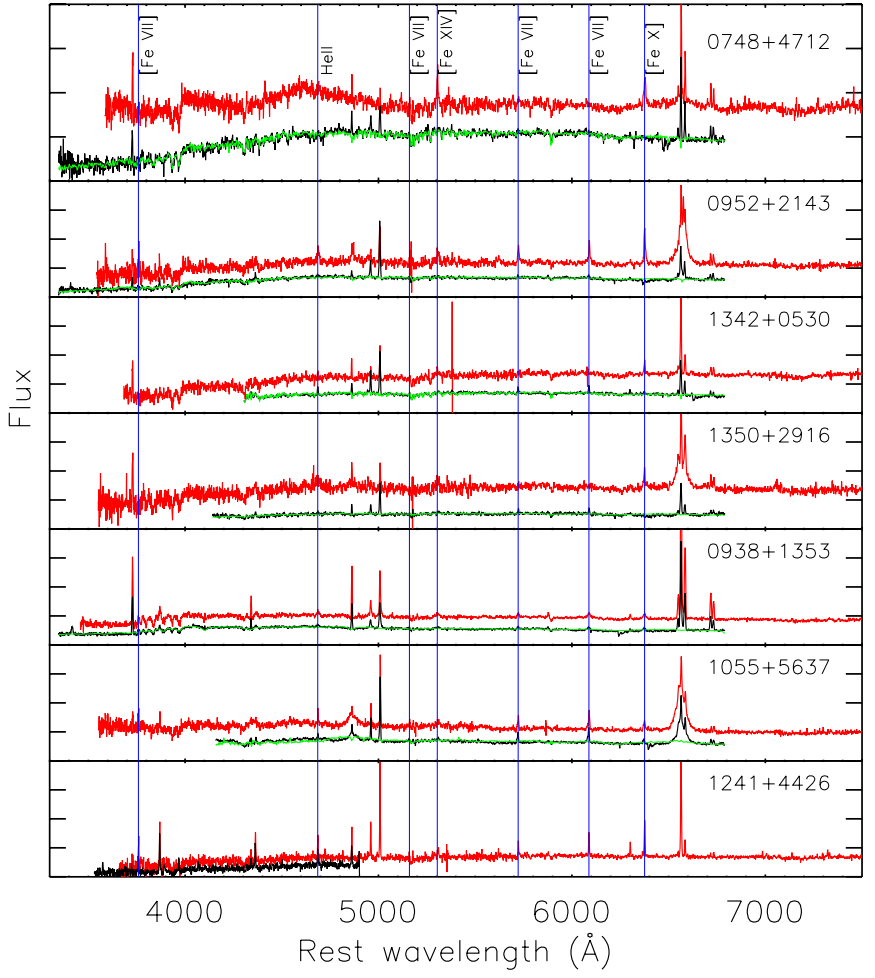

Figure 1. MMT (black) and SDSS (red) spectra of seven extreme coronal line emitters from W12. Continuum fits are plotted in green. Coronal lines and He II $\lambda 4686$ are marked in blue. The spectra have been shifted in the vertical direction for clarity.

(A color version of this figure is available in the online journal.)

however, that a significant fraction of the coronal lines come from the extended region. Therefore, the coronal lines will not be significantly affected by this aperture effect.

An initial check of Figure 1 suggests two different variability trends for coronal lines. In four of the seven objects, the high-ionization coronal lines [Fe x] $\lambda 6376-[\mathrm{Fe} X I V] \lambda 5304$ completely disappear in the MMT spectra, while for the other three objects $\mathrm{J} 0938+1353$, J1055+5637, and J1241+4426, the MMT spectra are similar to the SDSS spectra with respect to strong high-ionization coronal lines. For J1241+4426, although we failed to obtain the red part of the spectrum of the galactic center, we find that the [Fe VII] $\lambda 3759$ and $[\mathrm{Ne}$ III] $\lambda 3896$ lines are prominent and do not show significant variation in the blue part of the spectrum. Therefore, the latter three objects seem to be AGNs. The broad Balmer lines, Fe II lines, and the non-stellar continuum remain very prominent in $\mathrm{J} 1055+5637$, thus it is a Type 1 Seyfert galaxy. Note that J1055+5637 is the only object in W11 with narrow-line ratios located in the AGN regime in all three BPT diagrams. J0938+1353 shows fairly broad coronal and high-ionization lines, superimposed on strong, much narrower, low-ionization lines, which come clearly from star forming regions. We suspect that coronal lines and high-ionization lines are from an obscured AGN. Thus, it is likely that $\mathbf{J} 0938+1353$ is a composite of a Seyfert 2 nucleus with star forming regions but its true nature remains mysterious as coronal lines are not usually seen in composite-type galaxies. Alternatively, the coronal lines may be related to tidal disruption of a giant star by a massive black hole; this process produces a much longer lasting flare than does the tidal disruption of a main-sequence star. The latter scenario can be tested with future spectroscopic follow-up observations. We will focus only on the four variable objects in later analyses.

\subsection{Continuum Modeling and Variability}

In this subsection, we model the continuum and examine the contribution of potential non-stellar continua in the SDSS spectra. W12 found that four of the seven objects show flux variations between the SDSS photometric and spectral observations, including the AGN J1055+5637. The two objects without [Fe VII] lines were brighter during the spectroscopic observations than during the photometric observations, indicating that there is a non-stellar continuum in the J0748+4712 and $\mathrm{J} 1350+2916$ spectra; it is difficult to assess the case for $\mathrm{J} 0952+2143$. If a TDE is responsible for the continuum variability, any non-stellar continuum emission would be very weak after 5-9 yr even if it existed during the SDSS spectroscopic observations. Indeed, in all the MMT spectra, the continua are dominated by starlight. Therefore, the continua in the MMT spectra can be used as first-order templates for the stellar light in the SDSS spectra by ignoring the radial gradients of the stellar populations.

In order to obtain the stellar spectrum beneath the emission lines and beyond the MMT spectral coverage, we fit the MMT spectra with a combination of six independent component (IC) templates derived from stellar populations (Bruzual \& Charlot 2003) with Ensemble Learning Independent Component Analysis. Previous tests show that such an extrapolation is reliable (see Lu et al. 2006 for details). In practice, a continuum fit to a narrow wavelength range (4430 $\AA-7560 \AA)$ of the MMT spectrum for J1350+2916 can match its SDSS spectrum (wavelength coverage from $3700 \AA$ to $8500 \AA$ ) well. During the fitting, we masked all prominent emission lines and convolved the templates with a Gaussian kernel and shifted them in redshift to match the width and the centroid of the absorption lines. The fits with the six ICs are also shown in Figure 1.

Keeping in mind that different amounts of starlight fall within the apertures, we rescaled the starlight models of J0748+4712, $\mathrm{J} 0952+2143$, and $\mathrm{J} 1350+2916$ derived from the MMT spectra to match the SDSS spectra by minimizing the residuals in the stellar absorption lines. This process yields the fits shown in Figure 2. Overall, the stellar absorption lines in the SDSS spectra are fit very well, suggesting that this approach is feasible. A non-stellar continuum is required for only SDSS J0748+4712. This result provides an independent confirmation to the continuum variability analysis; furthermore, these findings also provide an estimate of the non-stellar continuum spectrum. Note this process is much less model-dependent than the spectral decomposition method using a combination of starlight plus a power-law or blackbody fit in W11. We describe the individual objects below.

For J0748+4712, its SDSS spectrum shows strong non-stellar emission and three broad bumps around 4050, 4600 and $6560 \AA$ (see W11). The scaling factor of the starlight in the SDSS spectrum is 1.35. After subtracting the stellar light spectrum, the differential spectrum is a smooth continuum plus several broad bumps and narrow emission lines (Figure 2). One notable difference from W11 is that there is no broad feature around $4050 \AA$, suggesting it was due to imperfect subtraction of stellar light in W11. The continuum is blue, but there is also a hint of curvature in the non-stellar spectrum. The latter is consistent with the variability analysis in W12 that the amplitudes appears larger in the $g$ - and $i$-bands than in the $r$-band. But we cannot rule out the possibility that there is an additional old stellar 


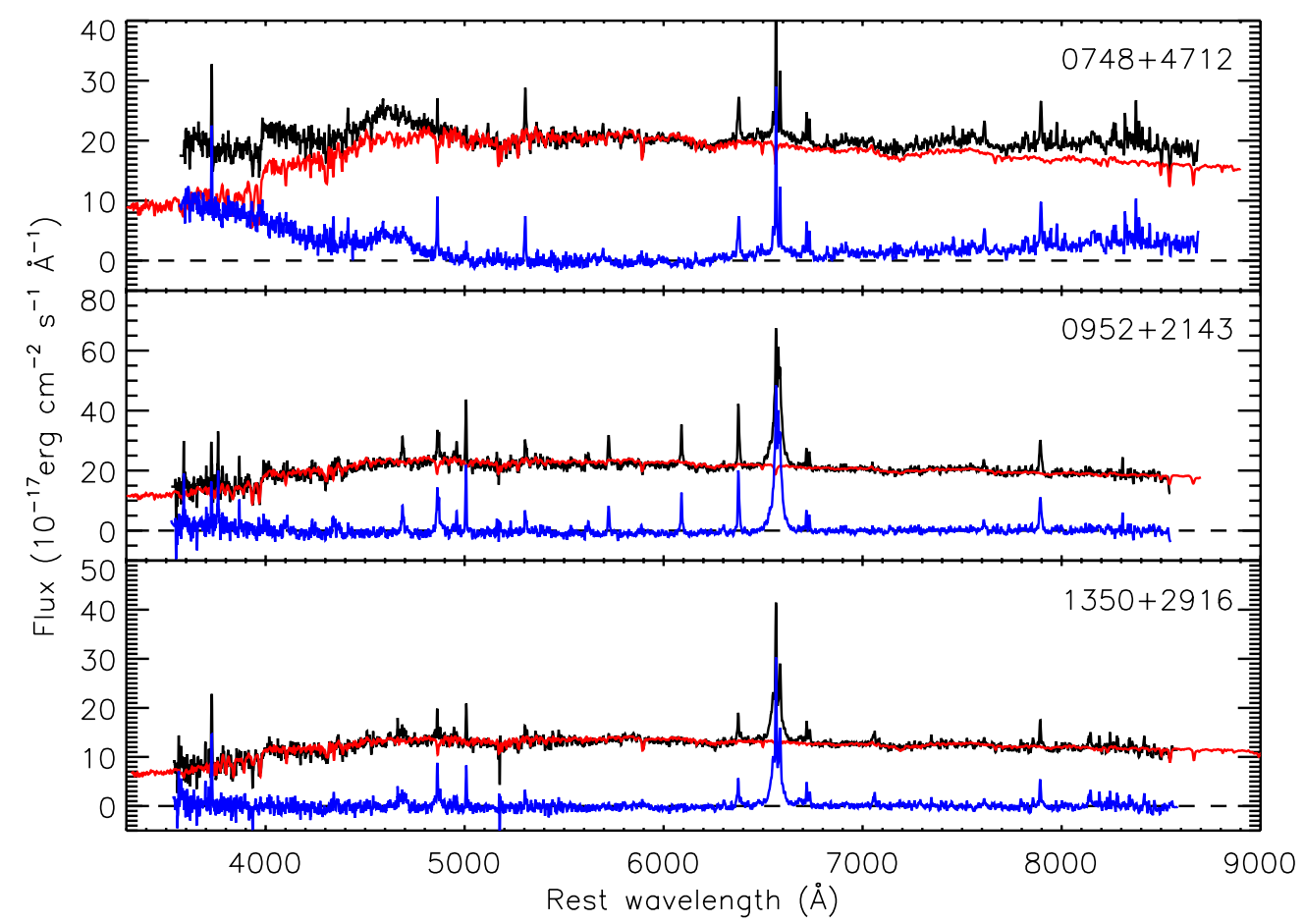

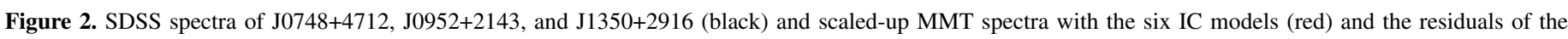
subtraction (blue). Both the spectra and the models are smoothed by three pixels.

(A color version of this figure is available in the online journal.)

population in the SDSS spectrum. If the curvature is confirmed, the non-stellar continuum may consist of two components, e.g., a disk component and a reprocessed outflow component.

It was reported that $\mathrm{J} 0952+2143$ also has a non-stellar continuum in its SDSS spectrum (Komossa et al. 2009). These authors fit the SDSS spectrum with a single simple stellar population plus blackbody emission. Our analysis does not confirm this. The SDSS continuum can be fit very well with a scaled-up MMT spectrum; therefore, a non-stellar component is not needed. For J1350+2916, W12 found that there was $3 \sigma$ evidence for brightening in the $g$-band between the SDSS photometric and spectroscopic observations. Our analysis does not require a non-stellar component in this case. However, for this object, the MMT spectrum does not cover the $4000 \AA$ break or blueward of it; this wavelength coverage is crucial for the detection of a non-stellar component because a nonstellar continuum is most prominent in shorter band and the $4000 \AA$ break provides strong constraints on the scale of the stellar component.

\subsection{Broad Emission Line Variability}

Three of the four objects displayed prominent broad $\mathrm{H} \alpha$, $\mathrm{H} \beta$, or He II $\lambda 4686$ lines in their SDSS spectra. Broad Balmer emission lines in J0952+2143 and J1350+2916 displayed double horns with separations between 2000 and $3000 \mathrm{~km} \mathrm{~s}^{-1}$ (see Komossa et al. 2008; W12). The FWHMs of these lines are 2100 and $2600 \mathrm{~km} \mathrm{~s}^{-1}$, respectively. J0748+4712 showed two strong and broad (several hundred $\AA$ ) bumps peaked around 4600 and $6560 \AA$. The broad bump at $4600 \AA$ was interpreted as blueshifted He II by W11. With the more reliable starlight subtraction here, this line also seems double peaked with the red peak close to the rest frame of He II while the blue peak is at $-8000 \mathrm{~km} \mathrm{~s}^{-1}$, although the signal-to-noise ratio $(\mathrm{S} / \mathrm{N})$ is still a bit low. It is hard to attribute the blue peak to the contamination of another broad line as there are no strong lines expected in this wavelength range. Although double peaks are not evident in the broad $\mathrm{H} \alpha$ line due to its weakness, the data are consistent with this result. Thus, broad lines in all three TDE candidates show double peaked profiles. We fit the narrow $\mathrm{H} \alpha$ and [N II] double lines with three narrow Gaussians and used a broad Gaussian to estimate the broad component of the $\mathrm{H} \alpha$ line. $\mathrm{H} \alpha$ is detected with a flux of $2.1 \times 10^{-15} \mathrm{erg} \mathrm{cm}^{-2} \mathrm{~s}^{-1}$. This value results in an $\mathrm{He}$ II $/ \mathrm{H} \alpha$ ratio of 3.3.

The broad bumps in J0748+4712 and the double horns in J0952+2143 and J1350+2916 disappeared in the MMT spectra (Figure 3). Only very weak broad $\mathrm{H} \alpha$ and $\mathrm{H} \beta$ lines can be seen in the MMT spectrum of J0952+2143 (middle panel of Figure 3 and Figure 4). Any broad lines, if present, must be below the detection limits of the other two objects.

To measure the broad emission lines in $\mathrm{J} 0952+2142$, we fit the continuum-subtracted spectra using Gaussians (Figure 4). All narrow lines show symmetric profiles. The $\mathrm{H} \alpha$ and $\mathrm{H} \beta$ lines display an additional redshifted broad component. Each symmetric narrow line was fit with a Gaussian, while one more Gaussian components were added for the redshifted broad component of each Balmer line. $\mathrm{H} \alpha$ and $\mathrm{H} \beta$ were fit with the widths and centroids of each component locked. The line fluxes are listed in Table 2. The broad component is redshifted by about $120 \mathrm{~km} \mathrm{~s}^{-1}$ relative to the systematic velocity and has an FWHM of $620 \pm 45 \mathrm{~km} \mathrm{~s}^{-1}$. This component could be the relic of the fading broad Balmer lines seen in the SDSS and New Technology Telescope (NTT) spectra that were described in Komossa et al. (2008, 2009). In those two spectra, which were taken on 2005 December 30 and 2008 February 6, respectively, the broad Balmer components had redshifts of $560 \mathrm{~km} \mathrm{~s}^{-1}$ and $270 \mathrm{~km} \mathrm{~s}^{-1}$, and FWHMs of $2100 \mathrm{~km} \mathrm{~s}^{-1}$ and $1500 \mathrm{~km} \mathrm{~s}^{-1}$, respectively. The line flux in the MMT spectrum is only $9 \%$ of that in the SDSS spectrum and $25 \%$ of that in the NTT spectrum. 

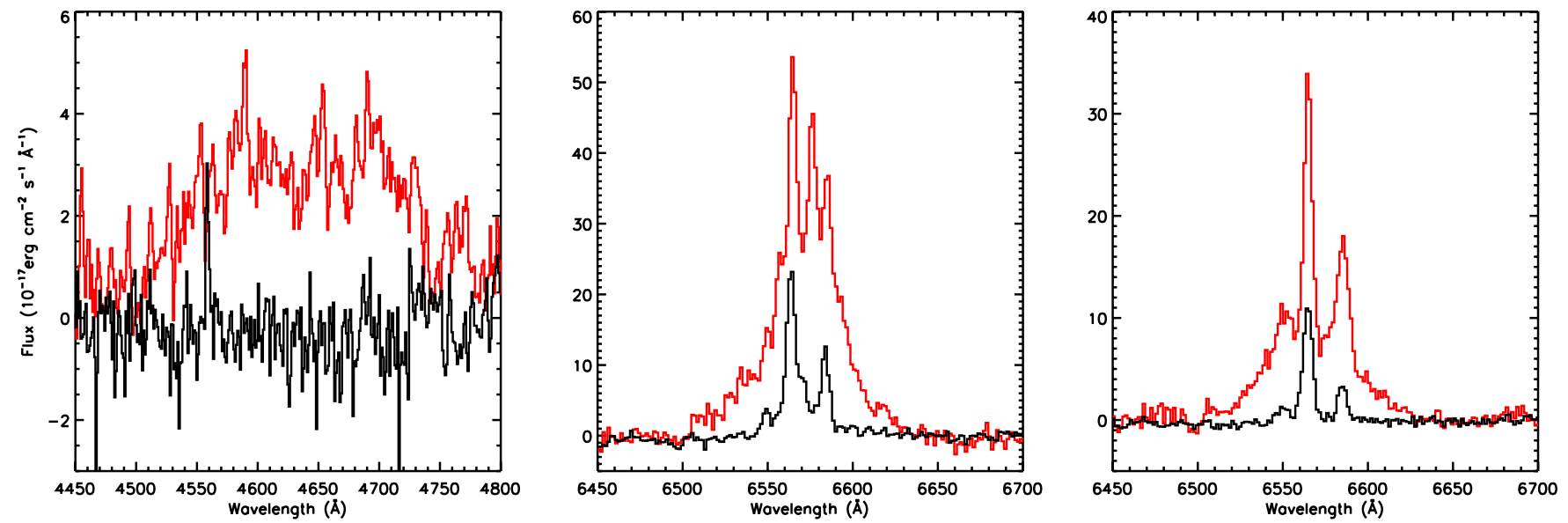

Figure 3. Broad bump around $4600 \AA$ in J0748+4712 (left panel; smoothed by three pixels) and H $\alpha$ regions of J0952+2143 (middle panel) and J1350+2916 (right panel). The MMT spectra are plotted in black and the SDSS spectra are plotted in red; all of the spectra have been continuum subtracted.

(A color version of this figure is available in the online journal.)

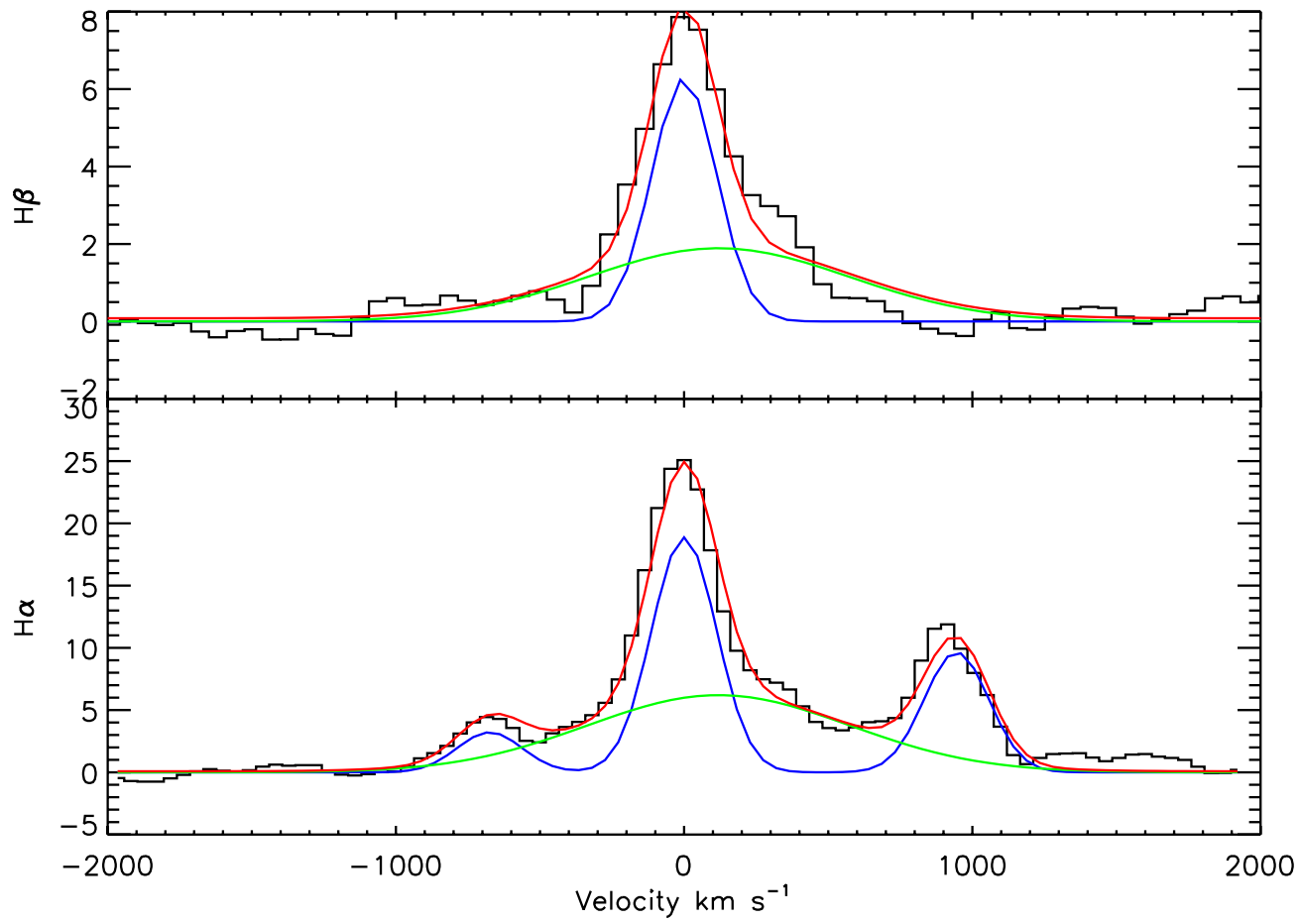

Figure 4. J0952+2143's H $\alpha$ (bottom panel) and H $\beta$ (top panel) line profiles (black) in the MMT spectrum. The normal narrow component fit is plotted in blue and the redshift broad component is plotted in green. The model sum is plotted in red.

(A color version of this figure is available in the online journal.)

Table 2

Narrow Emission Line Flux of Variable Targets ${ }^{a}$

\begin{tabular}{|c|c|c|c|c|c|c|c|c|c|c|c|c|c|c|c|}
\hline No. & $\mathrm{H} \alpha^{\mathrm{n}}$ & $\overline{\mathrm{H}} \beta^{\mathrm{n}}$ & $\mathrm{H} \alpha^{\mathrm{b}}$ & $\mathrm{H} \beta^{\mathrm{b}}$ & $\begin{array}{c}\mathrm{Ne} \text { III }] \\
\lambda 3896\end{array}$ & $\begin{array}{l}{[\mathrm{He} \mathrm{II}]} \\
\lambda 4686\end{array}$ & $\begin{array}{c}{\left[\mathrm{N}_{\mathrm{II}}\right]} \\
\lambda 6583\end{array}$ & $\begin{array}{c}{[\mathrm{OI}]} \\
\lambda 6300\end{array}$ & $\begin{array}{l}{[\mathrm{O} \text { III] }} \\
\lambda 4363\end{array}$ & $\begin{array}{l}{[\mathrm{O} \text { III] }} \\
\lambda 4959\end{array}$ & $\begin{array}{l}{[\mathrm{O} \text { III] }} \\
\lambda 5007\end{array}$ & $\begin{array}{c}{[\mathrm{Fe} \text { VII }]} \\
\lambda 5722\end{array}$ & $\begin{array}{c}{[\mathrm{Fe} \text { VII }]} \\
\lambda 6088\end{array}$ & $\begin{array}{c}{\left[\mathrm{S}_{\mathrm{II}}\right]} \\
\lambda 6716\end{array}$ & $\begin{array}{c}{[\mathrm{S} \mathrm{II}]} \\
\lambda 6731\end{array}$ \\
\hline 1 & 131(4) & $49(4)$ & & & $<2$ & $<6$ & $48(4)$ & $12(3)$ & $<8$ & $31(4)$ & $65(4)$ & $<3$ & $<4$ & $23(4)$ & $21(4)$ \\
\hline 2 & $108(7)$ & $26(4)$ & $155(10)$ & $39(7)$ & $31(4)$ & 11(3) & $64(5)$ & $14(4)$ & $<16$ & $72(3)$ & $215(3)$ & 13(3) & $17(5)$ & $25(4)$ & $27(4)$ \\
\hline 4 & $79(5)$ & $15(3)$ & & & & $6(2)$ & $22(4)$ & $<5$ & $<9$ & $22(1)$ & $66(1)$ & $8(2)$ & $12(4)$ & $9(2)$ & $9(2)$ \\
\hline
\end{tabular}

Note. a Emission line fluxes are in units of $10^{-17} \mathrm{erg} \mathrm{cm}^{-2} \mathrm{~s}^{-1}$. A superscript " $\mathrm{n}$ " means narrow line components and a superscript "b" means broad line components. 


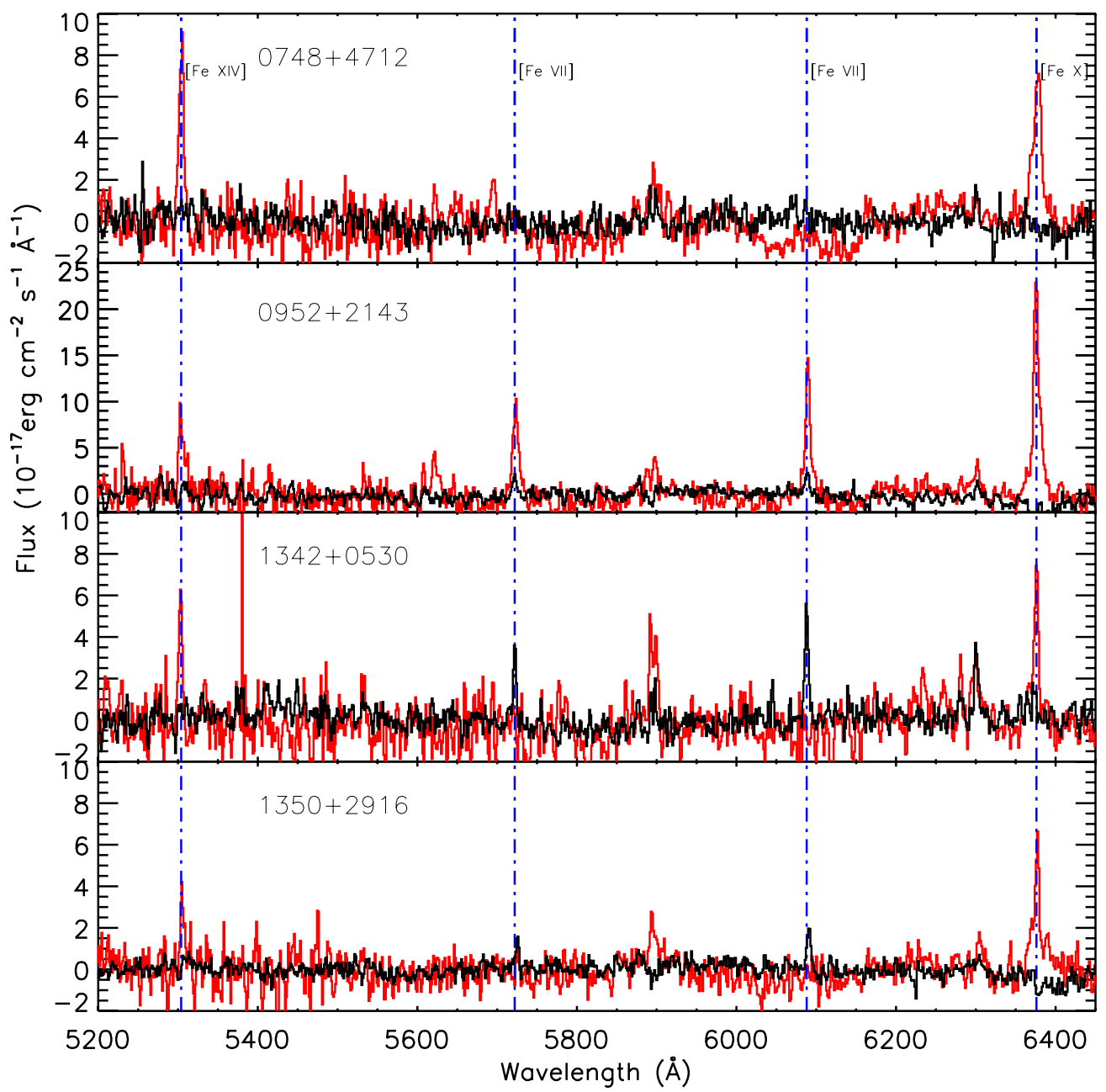

Figure 5. Coronal line of four TDE targets. From top to bottom: J0748+4712, J0952+2143, J1342+0530, and J1350+2916 (the MMT spectra are shown in black and the SDSS spectra are shown in red).

(A color version of this figure is available in the online journal.)

\subsection{Coronal Line Variability}

Figure 5 compares the coronal line spectra after subtracting the continuum of the MMT spectra from the SDSS spectra over the wavelength range $5200 \AA$ to $6450 \AA$. This wavelength coverage includes the coronal lines [Fe VII], [Fe X] $\lambda 6376$, and $[\mathrm{Fe}$ XIV] $\lambda 5304$. The one Gaussian fit results are also listed in Table 2. A close look at Figure 5 reveals some interesting variation patterns. Two of three objects without [Fe VII] emission lines in the SDSS spectra now show these lines in the MMT spectra, (J1342+0530 and J1350+2916). In J0952+2142, [Fe VII] lines prominent in the SDSS and NTT spectra have now become very weak. However, individual objects also show some different properties, as will be further described below.

J0748+4712. Its SDSS spectrum shows strong highionization coronal lines ([Fex], [Fe XI], and [Fe XIV]) but no [Fe VII] lines. In follow-up spectroscopic observations in 4-5 yr after the SDSS observations, all the coronal lines disappeared although the $\mathrm{S} / \mathrm{Ns}$ of the spectra were very low (W11). The high-S/N MMT spectrum confirmed this result; there were no [Fe VII] or higher ionization lines. These findings mark this object as having the shortest duration coronal lines.

$J 0952+2143$. Its SDSS spectrum shows both high-ionization coronal lines and relatively low-ionization coronal lines
([Fe VII]). In follow-up observations carried out with the Xinglong $2.16 \mathrm{~m}$ telescope and the NTT 2-3 yr later, all the coronal lines with ionizations higher than [Fe VII] disappeared and the [Fe VII] lines showed a marginal decrease (Komossa et al. 2008, 2009). In our MMT spectrum, only weak [Fe vII] lines are detected.

$J 1342+0530$. Its SDSS spectrum shows strong [Fe x], [Fe XI], and [Fe XIV] but no [Fe VII]. In the MMT spectrum, [Fe X], [Fe XI], and [Fe XIV] disappear but [Fe VII] lines are present.

$J 1350+2916$. The variability of the coronal line spectrum is similar to that of $\mathrm{J} 1342+0530$.

In summary, for all these sources, the coronal lines shift from high- to low-ionization species, or to a lack of coronal lines.

\subsection{Other Narrow Emission Lines}

W12 showed that conventional narrow-line ratios measured from SDSS spectra place these objects in the locus of starforming galaxies on the BPT diagram (Baldwin et al. 1981; also Kewley et al. 2006). This suggests that these lines come mainly from star-forming regions around the nuclei. Figure 6 shows the line ratios measured from the MMT spectra on the BPT diagrams. The line ratios are now very close to or above the line of demarcation between extreme AGNs and the star forming 


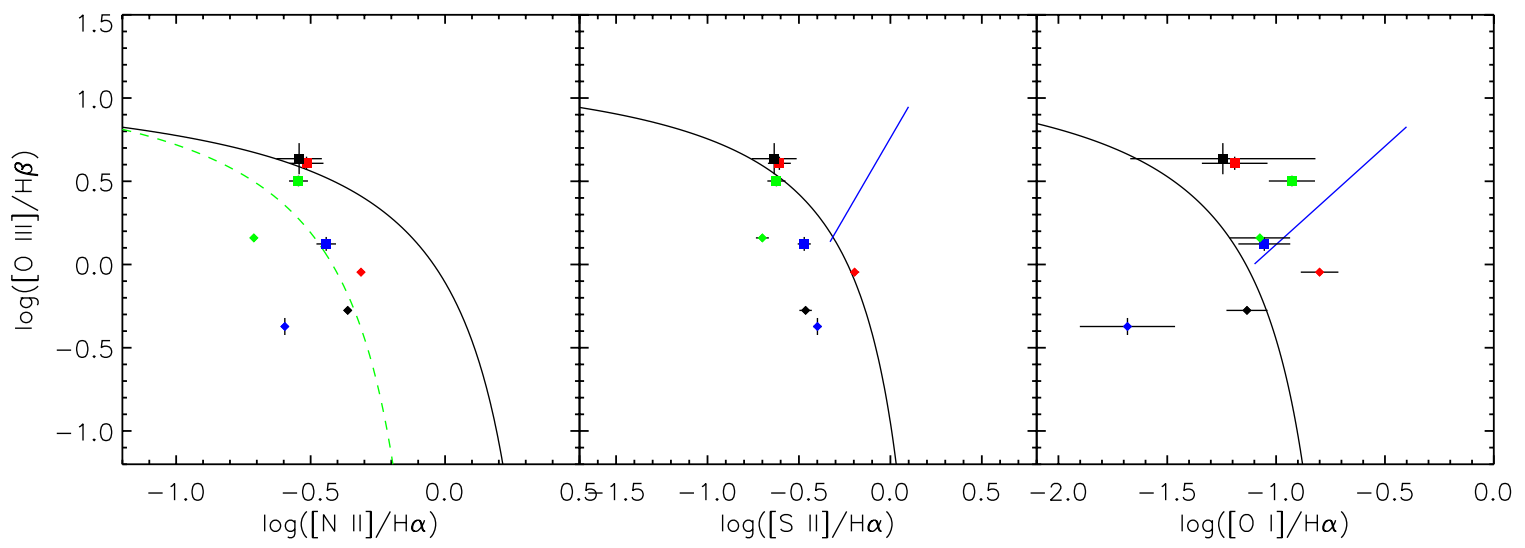

Figure 6. Evolution of TDE targets in BPT diagrams (diamonds: SDSS; squares: MMT; blue: J0748+4712; red: J0952+2143; green: J1342+0530; black: J1350+2916). The targets evolve from the star-forming region toward the extreme star-forming region and the weak Seyfert region. The changes are mainly due to the increasing of [O III] line strength and the smaller aperture of the MMT spectra.

(A color version of this figure is available in the online journal.)

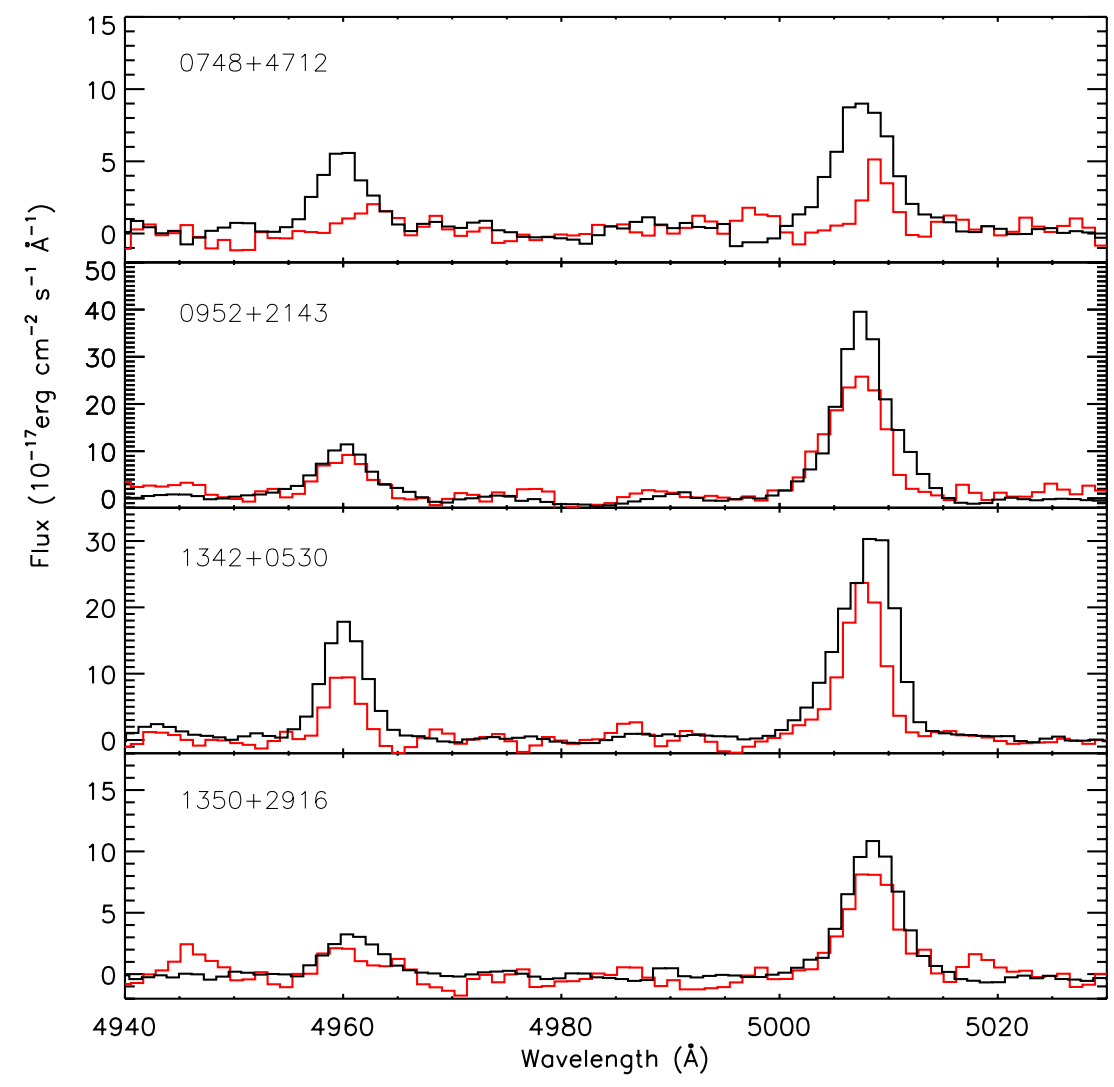

Figure 7. [O III] lines of the four TDE targets. Same order and color as in Figure 5.

(A color version of this figure is available in the online journal.)

region. This result is at least partially attributed to the increase of $[\mathrm{O}$ III] from the nucleus, as discussed below.

Changes in the BPT diagram must be interpreted with caution because the SDSS and MMT spectra were obtained using different apertures. The MMT long-slit spectra are extracted from a smaller aperture $\left(1^{\prime \prime} \times 3^{\prime \prime}\right)$ than the SDSS fiber size ( $3^{\prime \prime}$ diameter circle), and thus contain less light of the host galaxy. The emission lines from the extended star forming regions should be weaker in the MMT spectra than in the SDSS spectra. Indeed, the low-ionization lines, including [S II], [N II], and narrow $\mathrm{H} \alpha$ and $\mathrm{H} \beta$, are substantially weaker in the MMT spectra than in the SDSS spectra (Table 2), suggesting that they come from a larger region. However, [O III] in the MMT spectra is significantly stronger than in the SDSS spectra (Figure 7 and Table 2). The differences $(360 \%, 33 \%, 75 \%$, and $50 \%$ for J0748+4712, J0952+2143, J1342+0530, and J1350+2916), respectively, are larger than the calibration uncertainty (typically $4 \%$ for the SDSS spectra and $<5 \%$ for the MMT spectra). This result suggests that [O III] $\lambda 5007$ has brightened since the SDSS observations. The increase must come from the very nuclear region that is related to the TDE.

It is surprising that we measure an [O III] doublet ratio $\left[\mathrm{O}_{\mathrm{III}}\right] \lambda 4959 /[\mathrm{O}$ III $] \lambda 5007 \simeq 1 / 2$, in $\mathrm{J} 0748+4712$ and $\mathrm{J} 1342+0530$, which is very different from the canonical value 


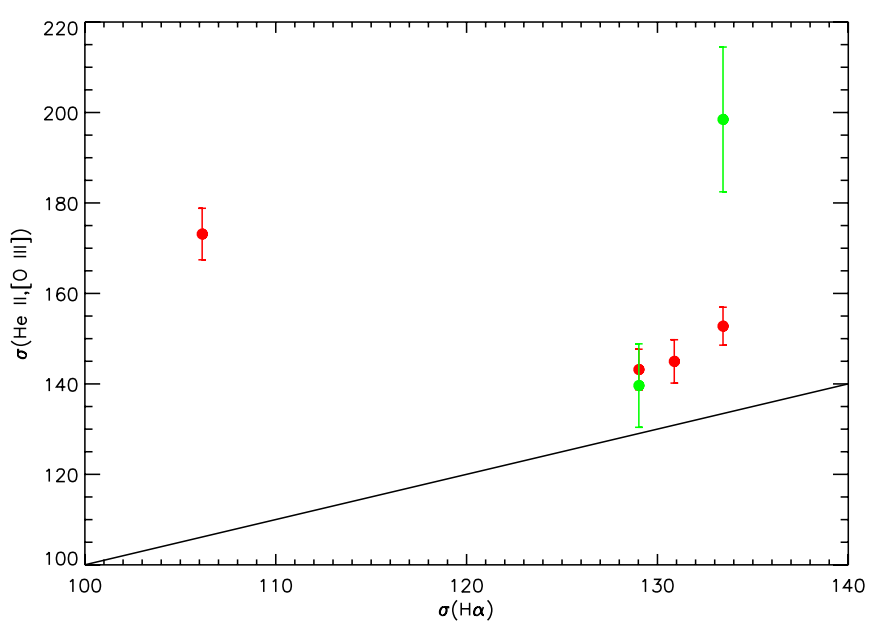

Figure 8. $\mathrm{H} \alpha$ line width vs. [O III] line width (red) and He II (green) line for the four TDE targets using the MMT spectra. Only lines with $>3 \sigma$ detections are plotted. The straight line denotes the one-to-one relation.

(A color version of this figure is available in the online journal.)

of 1/3 (Figure 7). To check for potential contamination from other lines, we examined the NIST atomic database for lines within $3 \AA$ of $\left[\mathrm{O}\right.$ III] $\lambda 4959 .^{6}$ Only Ti I $\lambda 4958.3$, Fe II $\lambda 4958.2$, $\mathrm{Pm} \mathrm{I} \lambda 4959.5$, and $\mathrm{Fe} \mathrm{I} \lambda 4961.2$ are within $3 \AA$ of $[\mathrm{O}$ III] $\lambda 4959$. Fe II can be ruled out because it would produce much stronger emission at $4889.6 \AA$ from the same upper level, which is not detected. For a similar reason, Fe I can be excluded because it predicts a stronger emission line at $4916.3 \AA$. Ti I and Pm I have the same problem in addition to very small elemental abundances. Therefore, contamination from other lines can be ruled out. We also looked for potential telluric absorption lines and sky lines around the redshifted line wavelengths and found none. Since [O III] $\lambda 4959$ and [O III] $\lambda 5007$ share the same upper level, the different line ratios can only be explained by radiative transfer effects, which require a substantial optical depth for the line. A detailed interpretation of this process is given in Section 4.2.

Narrow He II $\lambda 4686$ shows a more complicated variability pattern. In J0952+2143, strong, narrow He II $\lambda 4686$ seen in the SDSS spectrum becomes barely detectable in the MMT spectrum, along with a weakening of the coronal lines and the broad lines. On the other hand, narrow He II $\lambda 4686$ remains unchanged or shows a small increase in $\mathrm{J} 1342+0530$ in the course of the decrease of high-ionization coronal lines and the appearance of [Fe VII]. The situation is not clear in the other two objects because of either blending with the broad component in the SDSS spectra or weakness of the line in the MMT spectra. Note that He II $\lambda 4686$ in both the SDSS and MMT spectra is systematically broader than other lowionization narrow emission lines, such as $\mathrm{H} \alpha$ and [S II], which mainly come from star-forming regions (Figure 8). This is consistent with the expectation that He II $\lambda 4686$ is powered mainly by the continuum flare, with little contribution from star formation.

\section{DISCUSSION}

\subsection{Formation and Evolution of the Broad Lines}

In our sample, three targets $(\mathrm{J} 0748+4712, \mathrm{~J} 0952+2143$, and $\mathrm{J} 1350+2916)$ show broad recombination lines $(\mathrm{H} \alpha, \mathrm{H} \beta$, or

\footnotetext{
6 Giving the similar profiles of the [O III] doublet in these two objects, contamination by lines with larger offsets relative to [O III] $\lambda 4959$ can be ruled out.
}

He II $\lambda$ 4686) in their SDSS spectra and all of these lines are double-peaked or strongly blue shouldered. Such lines can be formed in biconical outflows launched by a super-Eddington accretion flow, in a fast outflowing unbound stellar debris, or in a ring of accreted debris; these materials photoionized by strong radiation from the accretion disk. Bogdanović et al. (2004) showed that photoionized debris produces only a weak broad $\mathrm{H} \alpha$ line $\left(L(\mathrm{H} \alpha) \sim 10^{37} \mathrm{erg} \mathrm{s}^{-1}\right)$, which is much smaller than the observed one, for the tidal disruption of a solar-type star by a black hole of mass $10^{6} M_{\odot}$. This result is because the stellar debris is confined to subtending only a small solid angle on the accretion disk. There are several possible ways in which emission lines can be enhanced. First, if the star is evolved, e.g., a subgiant, the debris may spread out more in the vertical direction because an evolved star has a much larger size and thus produces more reprocessed emission lines. Second, if the star's orbit misaligns with the spin direction of the black hole, the debris disk may receive more light from the accretion disk. Finally, the line equivalent width is likely also dependent on the mass of the black hole. Small black holes may produce larger line equivalent widths because the tidal disruption radius is smaller so the stellar debris has a larger covering factor. It remains to be seen whether taking into account all these effects can reproduce the observed line equivalent width. Thus, we favor the biconical outflow model because it predicts a large line equivalent width and a double-peak profile, both of which are more consistent with observed lines (Strubbe \& Quataert 2009).

In either scheme, broad lines are expected to fade on the timescales of months to a year. The fading is caused by both the declining of the ionizing continuum and the evolution of the debris properties or outflows. The variation of the ionizing continuum is qualitatively understood, a nearly constant luminosity in the super-Eddington phase and a power-law decay phase with an index around $-5 / 3$ to $-5 / 2$ (Rees 1988; Lodato et al. 2009). In either stage, the ionizing continuum becomes softer. In a quasi-steady accretion system, it is expected that the outflow weakens as the accretion rate decreases. Since the accretion rate varies on dynamic timescales, the structure of the outflow is likely complicated. The disk initially launches strong outflows during the super-Eddington phase, and the outflows are accelerated to high velocities. As the accretion rate decreases, the disk will produce a weak outflow and the radiation acceleration is greatly reduced; after a certain time, the radiation acceleration may eventually become smaller than the gravitational one, and then the outflow decelerates in the gravitational field.

In J0748+4712 and J1350+2916, we can only put upper limits on the lifetime of the broad lines to $5 \mathrm{yr}$. In J0952+2143, we witness the declining of the broad lines. In the NTT spectrum taken 768 days after the SDSS spectrum, the redshifted broad Balmer emission lines and double horn profile are still prominent (Komossa et al. 2009). The line flux declined by a factor of only 2.8 in comparison with the SDSS observations. This decline is slower than a $\propto t^{-4 / 3}$ or $t^{-5 / 3}$ law if we take the initial flare to have occurred temporally near the SDSS photometric observation, 375 days before the SDSS spectroscopic observation. In its MMT spectrum, which was taken 2187 days after the SDSS spectrum, the double horn profile disappeared but the weak redshifted broad component still exists. Taking into account the short recombination timescale of excited $\mathrm{H}$ atoms $\left(\approx 10^{5} / n_{e} \mathrm{yr}\right.$; Osterbrock \& Ferland 2006), an ionizing continuum is needed to produce the broad Balmer lines. The continuum may form through late-stage accretion of residual stellar debris in very 
eccentric orbits, or by fallback of failed outflows. The broad line flux is a factor of 4 lower than in the NTT spectrum. Noting that both the line width and the centroid offset decrease with time, this result is consistent with the decelerating outflow scenario.

Probably due to the relatively small size of the accretion disk, outflows from both sides are observed, leading to a double peaked profile. This profile is in contrast to the single-peak blueshifted profiles of C IV in luminous quasars (Richards et al. 2011; Wang et al. 2011). The He II line in J0748+4712 is not symmetrical around zero velocity in the source rest frame. This result perhaps can be attributed to the partial obscuration of the base of the receding outflow. The small separation between the two peaks in the other two objects can be produced by either a large inclination to the collimated outflows or a small outflow velocity. As noted in W12, J0748+4712 was observed by SDSS much earlier than the other two objects when the disk emission was still strong and outflows showed a high velocity. With the decrease of the accretion power, the outflows were decelerated in the gravitational potential of the black hole, leading to small outflow velocities in the other two objects.

As discussed in W11 (see also Peterson \& Ferland 1986; Gezari et al. 2012), the large $\mathrm{He} \mathrm{II} / \mathrm{H} \alpha$ ratio requires an overabundance of helium relative to hydrogen, which is interpreted by W11 as the tidal disruption of an evolved star. Following Peterson \& Ferland (1986) and assuming a gas temperature of $2 \times 10^{4} \mathrm{~K}$, a minimum of $n_{\mathrm{He}} / n_{\mathrm{H}}=0.75$ is required by assuming that most of helium is in the $\mathrm{He}^{+2}$ phase.

Most of the TDEs discovered by X-ray and UV flares do not show broad emission lines, while three of four objects in this sample do. This result may be attributed to selection effects. On the one hand, the broad line objects are usually considered to be AGNs and rejected for further monitoring to reduce the number of sources for repeated spectroscopic follow-up (Gezari et al. 2009; van Velzen et al. 2011; Cenko et al. 2012). On the other hand, coronal line-selected objects preferably lie in the gas-rich disk galaxies, and they tend to host small black holes. The accretion rate is expected to be higher than that of a highmass black hole, and outflows should be denser and stronger. If broad lines are formed in outflows, one would naturally expect that TDEs from small black holes would produce strong broad emission lines. More theoretical calculations are required to verify this hypothesis, however.

\subsection{Consequences of Optically Thick [O III] Emission}

\subsubsection{Simple Estimates}

The [O III] line ratio, which is $\sim 2$ rather than the expected canonical value 3 , suggests that the lines are optically thick. In this section, we examine some consequences of this observation. This discussion is preliminary because there are not yet sufficient spectroscopic constraints to fully define a model for the observations.

If a line is thermalized, that is, its density is substantially above the critical density, then the level populations are given by the gas kinetic temperature and line photons are lost in the scattering process because they are collisionally de-excited. The intensity of a line is then given by $I_{\nu}=B_{\nu}[1-\exp (-\tau)]$ where $B_{v}$ is the Planck function and $\tau$ is the line optical depth. The $\lambda \lambda 5007,4959$ line optical depths $\tau$ are in a $3: 1$ ratio, so the intensity ratio is given by $I(\lambda 5007) / I(\lambda 4959)=$ $[1-\exp (-\tau)] /[1-\exp (-\tau / 3)] \approx 2$. This function is shown in Figure 9 . A line ratio of $\approx 2$ corresponds to an optical depth $\tau(\lambda 5007) \approx 1.5$.

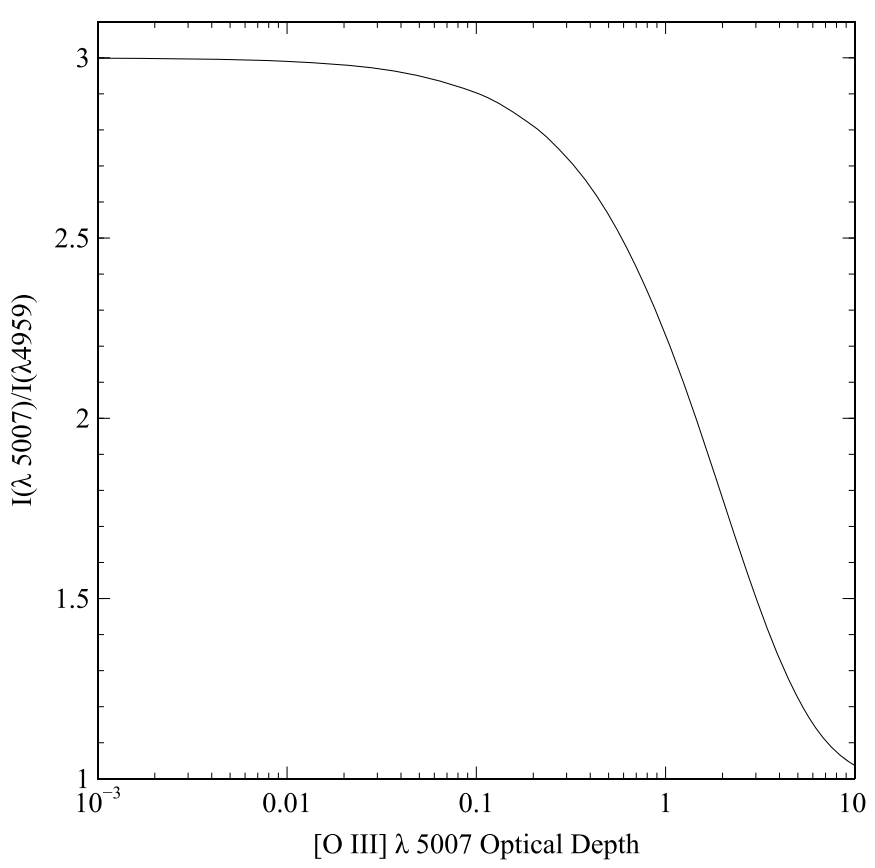

Figure 9. Dependence of the [O III $] \lambda 5007 / \lambda 4959$ ratio on the optical depth of $\lambda 5007$. A line ratio of $\approx 2$ corresponds to an optical depth $\tau(\lambda 5007) \approx 1.5$.

The actual line ratio is a function of both optical depth and density. If the electron density is well below the critical density of the ${ }^{1} \mathrm{D}_{2}$ level that produces the [O III] lines, then photons will be remitted after absorption. These photons will simply scatter out of the cloud with no loss of intensity and the 3:1 line ratio will be maintained. Line photons are only lost when there is a large probability of collisional de-excitation and they are thermalized following absorption. The lines will be well thermalized when the density is $n_{e} \gg n_{\text {crit }}\left({ }^{1} \mathrm{D}_{2}\right) \approx 6.8 \times 10^{5} t_{4}^{0.5} \mathrm{~cm}^{-3}$, where $t_{4}=T / 10^{4} \mathrm{~K}$. If the density is less than this value, a larger $\lambda 5007$ optical depth would be needed to reach the same line ratio.

More O III lines would be needed to estimate the parameters for the [O III]-forming region. A good detection of the $\lambda 4363$ line would be important. These observations are not available so we will simply assume that the $\lambda 5007$ optical depth is of the order of unity, $t_{4}=10^{4} \mathrm{~K}$, and that the electron density is of order the ${ }^{1} \mathrm{D}_{2}$ critical density.

\subsubsection{Column Density and Compton Depth}

The line absorption coefficient is given by

$$
\alpha=1.497 \times 10^{-6} f_{l u} \lambda \mu \mathrm{m} / u_{\text {Dop }} \mathrm{cm}^{2},
$$

where $f_{l u}$ is the absorption oscillator strength, $\lambda \mu \mathrm{m}$ is the wavelength in microns, and $u_{\text {Dop }}$ is the Doppler width in velocity units. Assuming the transition probabilities given by Storey \& Zeippen (2000), we find $\alpha=5.57 \times 10^{-17} u_{\text {Dop }}^{-1} \mathrm{~cm}^{2}$.

The Doppler width is given by the thermal width if turbulence is absent:

$$
\begin{aligned}
u_{\mathrm{th}}= & \sqrt{2 k T / m} \mathrm{~cm} \mathrm{~s}^{-1} \approx 12.8 \sqrt{t_{4} / m_{\mathrm{AMU}}} \mathrm{km} \mathrm{s}^{-1} \\
& \approx 3.2 t_{4}^{1 / 2} \mathrm{~km} \mathrm{~s}^{-1},
\end{aligned}
$$

where the expression is evaluated for oxygen, $m_{\mathrm{AMU}}=16$. The absorption cross section becomes

$$
\alpha=1.74 \times 10^{-22} t_{4}^{-1 / 2} \mathrm{~cm}^{2} .
$$


The column density required for $\tau(\lambda 5007)>1$ is then

$$
N\left(\mathrm{O}^{2+}\right)>5.75 \times 10^{21} t_{4}^{1 / 2} \mathrm{~cm}^{-2} .
$$

The solar $\mathrm{O} / \mathrm{H}$ ratio is $4.9 \times 10^{-4}$ (Asplund et al. 2009) so the total hydrogen column density at a metallicity $Z$ will be

$$
N(\mathrm{H})>1.2 \times 10^{25} t_{4}^{1 / 2} Z^{-1} \mathrm{~cm}^{-2} .
$$

The corresponding electron scattering optical depth is then

$$
\tau(e)=\sigma_{T} N(\mathrm{H})>7.8 t_{4}^{1 / 2} Z^{-1},
$$

where $\sigma_{T}$ is the Thomson cross section. This has the important consequence that, unless the metallicity is quite large $(Z \geqslant 10)$, the [O III] line-forming region is optically thick to electron scattering.

This result means that we may have underestimated the line width in Equation (2) since $\lambda 5007$ photons will scatter off the rapidly moving electrons. The mass of an electron is $2.94 \times 10^{4}$ smaller than that of an oxygen atom so the line width is 171 times wider. The column densities are increased by $\sqrt{171} \simeq 13$ if electron scattering dominates the line width.

The thermal width of [O III] $\lambda 5007$ at $10^{4} \mathrm{~K}$ would be $\sim 550 \mathrm{~km} \mathrm{~s}^{-1}$ in the electron-scattering-dominated limit. This value is substantially larger than the observed widths, 200 and $255 \mathrm{~km} \mathrm{~s}^{-1}, 7$ indicating that the lines are not broadened into the full electron scattering limit. At optical depth $\tau(e)<1$, the scattered photons will produce an extended wing with a width dependent on the optical depth and electron temperature, superimposed on the primary emission profile (Laor 2006). Thus, the profile of the high velocity wing can be used to constrain the properties of the scatter. Unfortunately, from our data we cannot confirm or rule out the existence of the broad wing of [O III] emission lines. The lack of a prominent wing would indicate that the gas has a high metallicity of the order of $Z \sim 10$.

\subsubsection{Suggestions from Photoionization Models}

The large column density suggested in Equation (5) places constraints on the radiation field shape and intensity. In its simplest form, the photoionization balance equation may be written as (Osterbrock \& Ferland 2006):

$$
\phi(\mathrm{H})=n_{e} n_{p} \alpha_{B} d l=n_{e} \alpha_{B} N(\mathrm{H}),
$$

where $\alpha_{B}$ is the hydrogen Case B recombination coefficient, $\phi(\mathrm{H})$ is the flux of hydrogen-ionizing photons, and $\mathrm{dl}$ is the Strömgren thickness, the thickness of the $\mathrm{H}^{+}$layer. This expression can be written in terms of the dimensionless ionization parameter $U$, the ratio of densities of hydrogen-ionizing photons to hydrogen:

$$
U(\mathrm{H}) \equiv \frac{\phi(\mathrm{H})}{n(\mathrm{H}) c}=1.1 \frac{\alpha_{B}}{c} N(\mathrm{H}),
$$

where we assume that helium is single ionized with a solar metallicity, so that $n_{e}=1.1 n(\mathrm{H})$. Inputting numerical values and assuming $\alpha_{B} \approx 2.6 \times 10^{-13} t_{4}^{-0.8}$, the limit in Equation (5) becomes

$$
U(\mathrm{H})>10^{2} t_{4}^{-0.3} Z^{-1} .
$$

\footnotetext{
7 This width is $\sqrt{2} \sigma_{v}$.
}

This is a large ionization parameter. Photoionization models of strong-[O III] lined objects are often fitted with $U \sim$ $10^{-2}-10^{-1}$. For photoionization by a continuum that extends to high energies, such as the spectral energy distribution (SED) of an AGN, the ionization of the gas is proportional to $U$ (Osterbrock \& Ferland 2006) and little $\mathrm{O}^{2+}$ is present for such large values.

This is not the case if the SED is soft. If few ionizing photons are present at energies that can ionize $\mathrm{O}^{2+}$ to $\mathrm{O}^{3+}$, then the required column density of $\mathrm{O}^{2+}$ will be produced. This occurs if the SED is equivalent to a blackbody with $T<5 \times 10^{4} \mathrm{~K}$, or if a more energetic SED is filtered through an intervening absorbing column that removes high-energy photons. The nature of the ionizing source is clearly a fundamental question for these objects.

A very large $U$ with a soft SED is consistent with the $10^{4} \mathrm{~K}$ temperature we have assumed. In photoionization equilibrium, the gas heating is proportional to the photoionization rate, which is equal to the recombination rate. There is no direct dependence on the flux of photons or on the ionization parameter. A stronger flux of photons produces higher ionization but the same photoionization rate, which is equal to the recombination rate, so the heating rate is constant.

\subsection{Formation and Evolution of Narrow Emission Lines}

Coronal lines and a significant fraction of [O III] lines must come from ambient gas photoionized by the flare as they are variable on timescales of several years. Most of the previous TDE candidates that were discovered via X-ray or UV flares did not have such features. The recently discovered TDE candidate PS1-10jh has a strong, broad He II $\lambda 4686$ line and weak Balmer lines as $\mathrm{J} 0748+4712$, but does not show coronal lines either (Gezari et al. 2012). Thus, only a fraction of TDEs show coronal lines, and the presence of coronal lines can be independent of the existence of broad lines. This can be understood in the framework already discussed. The presence of coronal lines is related to the gas distribution close to the supermassive black hole (W12), while the formation of broad lines depends on the strength of outflows or on the geometry of debris relatively close to the disk radiation, which is likely anisotropic. Although the nuclear gas environment and outflows are expected to be correlated with the black hole mass in the local universe, at least statistically, there are many outliers.

Komossa et al. (2008) postulated that coronal lines are echoes of the continuum flare by distant gas. As discussed in W12, the variations of emission lines are rather complex in this scenario, depending on the time evolution of the flare as well as on the distribution of the gas surrounding the massive black hole. The emission lines at a given time come from different regions that are illuminated by the continuum with different time advances. Thus, in the optically thin case, the optimal emission region for a specific line is determined by competition between the time fading of the continuum, the position-dependent time lag, the $r^{-2}$ dilution of the incident continuum intensity, and the radial dependence of the density. Figure 10 shows a snapshot of the ionization parameter distribution for a model in which the ionizing continuum varies with time as a declining power-law $L(t) \propto t^{-5 / 3}$ and the density distribution is smoothly varying: $n \propto r^{-\beta}$. In the optically thick case, additional frequencydependent attenuation has to be considered, which steepens the ionizing continuum in distant regions.

Even with sparse sampling, the observations clearly require a different duration timescale of coronal lines in different objects. 


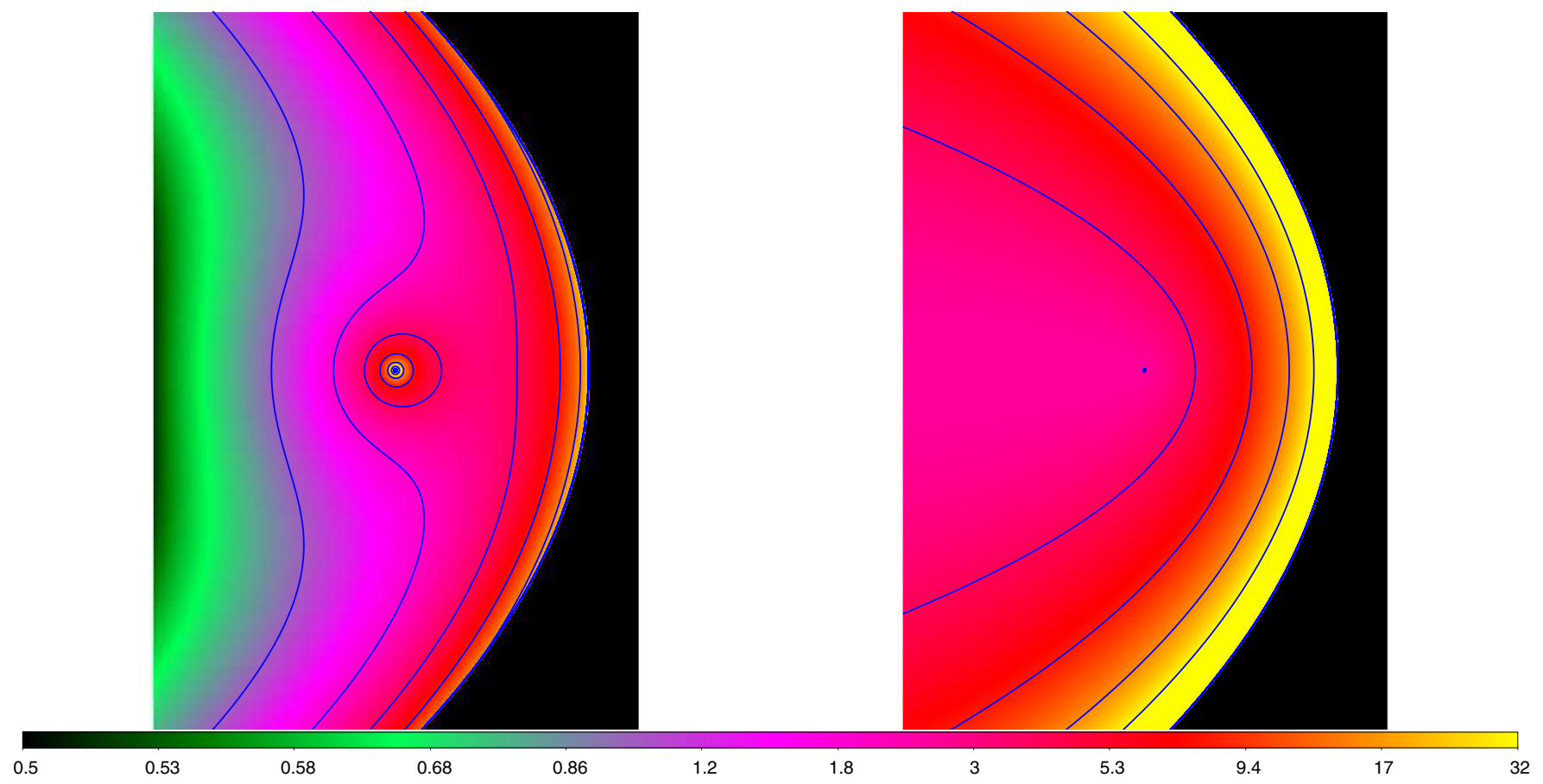

Figure 10. Snapshot of the ionization parameter around a black hole that contributes to the observed emission lines at a specific time in an oversimplified model, in which the ionizing continuum decreases with time as $\propto t^{-5 / 3}$ when $t>t_{0}$ (where $t_{0}$ is the time between when the star was tidally disrupted and when most of the bound material returned to the pericenter). The density distribution decreases as $\propto r^{-1}$ (left panel) and $\propto r^{-2}$ (right panel). The observer is to the left of the figure at time $10 t_{0}$. The overlaid blue lines are intensity contours with an interval of two time folds.

(A color version of this figure is available in the online journal.)

Coronal lines in $\mathrm{J} 1342+0530$ lasted for a timescale of at least $10 \mathrm{yr}$ with a transition from high-ionization coronal lines to lowionization lines only, while in J0748+4712, all coronal lines disappeared in fewer than 4-5 yr. It is not clear what causes the different timescales: the continuum light curve or the gas distribution. We do not know what happened between the SDSS observations in 2004 and the Xinglong $2.16 \mathrm{~m}$ observations in 2009 for J0748+4712. Was there a similar transition from highionization coronal lines only to low-ionization coronal lines, or did all coronal lines disappear simultaneously? Furthermore, also for $\mathrm{J} 1342+0530$ and $\mathrm{J} 1350+2916$, is there an intermediate state with both high- and low-ionization coronal lines, as seen J0952+2143? If so, then there is a continuous transition from high- to low-ionization state and we can unify the high- and low-ionization coronal line emitter through time evolution. Unfortunately, we do not have the data in these important evolutionary stages.

[O III] variability provides additional constraints on the gas distribution in the inner parsecs and the evolution of the ionizing continuum. The brightening of [O III] follows the declining of coronal lines, i.e., the average ionization of line-emitting gas decreases continuously. Giving the short recombination times of $\mathrm{O}^{2+}\left(1.3 \times 10^{5}\left(10^{6} \mathrm{~cm}^{-3} / n_{e}\right) \mathrm{s}\right)$, the gas is in quasi-ionization equilibrium. This requires either attenuation of the ionizing continuum or/and the density must decrease more slowly than $r^{-2}$. The analysis in the last section suggests a soft ionizing continuum. Combined with the strong coronal line emission in the early spectra, these results support the idea that the hard ionizing photons have already been filtered.

In the last section, we derived a lower limit on the gas column density for two sources (J0748+4712 and J1342+0530) with non-canonical $\left[\mathrm{O}_{\mathrm{III}}\right]$ ratios. Bear in mind that the flare lasts fewer less than $10 \mathrm{yr}$, so the $\mathrm{O}^{2+}$ ions exist within the region bounded by the parabolic surface with a lag of $10 \mathrm{yr}$ (see figure in W12). The large column density sets a lower limit on the gas density of the order of $n(\mathrm{H}) \sim 1.2 \times 10^{25} t_{4}^{1 / 2} Z^{-1} / c \Delta t \mathrm{~cm}^{-3}=$ $2 \times 10^{6} t_{4}^{1 / 2} Z^{-1} \Delta t_{6}^{-1} \mathrm{~cm}^{-3}$, where $\Delta t_{6}=(\Delta t / 6) \mathrm{yr}$.

Assuming that all observed [O III] comes from the same region, we can derive the volume and mass of the [O III] emitting gas. At a temperature of $10^{4} \mathrm{~K}$, the volume emissivities of [O III] $\lambda 5007$ are $2.0 \times 10^{-16}, 1.7 \times 10^{-14}, 6.7 \times 10^{-13}$, and $9.5 \times 10^{-12} \mathrm{erg} \mathrm{s}^{-1} \mathrm{~cm}^{-3}$ for $n(\mathrm{H})=10^{4}, 10^{5}, 10^{6}$, and $10^{7}$ $\mathrm{cm}^{-3}$, respectively, assuming a solar abundance. The observed [O III $] 25007$ luminosities for J0748+4712 and J1342+0530 are similar and around $9 \times 10^{39} \mathrm{erg} \mathrm{s}^{-1}$, requiring a volume of line-emitting gas of only $2.7 \times 10^{52} \mathrm{~cm}^{3}$ for $n(\mathrm{H})=10^{6} \mathrm{~cm}^{-3}$. This volume is only a small fraction of the volume swept by the flare radiation in nearly $10 \mathrm{yr}$, suggesting a small filling factor. Combining this result with a large column density implies that the emission region consists of only a small number of thick clouds.

\subsection{Could the Power Source be AGN Variability?}

A substantial fraction of AGNs show coronal lines, but their strengths are usually much weaker than that of [O III]. In the SDSS spectra, coronal lines of these TDE candidates are 1-2 orders of magnitude stronger than the strong coronal lineemitting Seyfert galaxies. Furthermore, a large body of AGN monitoring has shown that coronal lines in most AGNs are quite stable, while in rare cases the coronal lines do vary on timescales of years and the amplification is usually moderate (Penston et al. 1984; Veilleux 1988). Only in one extreme case (IC 3599) was a strong decline of $[\mathrm{Fe} \mathrm{x}]$ by two orders of magnitudes observed after an unusual soft X-ray burst; this event has sometimes been interpreted as a TDE (Brandt et al. 1995; Grupe et al. 
1995; Komossa \& Bade 1999). In our four TDE targets, coronal lines with ionization potentials higher than that of [Fe VII] show strong fading of more than two orders of magnitude within $10 \mathrm{yr}$ and [Fe VII] lines appear in two targets that lack them in the SDSS spectra. That makes them very extreme if they were AGNs. In addition, large amplitude variations (a factor of 100 ) in the highly ionized coronal lines require large amplitude variations in soft X-rays. Up until now, only narrow line Seyfert 1 galaxies and BL Lac objects have been known to show such large amplitude variability in X-rays (Eracleous et al. 2012). All seven ECLE targets were covered by the FIRST survey (Becker et al. 1995), but only J0938+1353 was detected with a low flux of $1.93 \mathrm{mJy}$, while others were below the detection limit of typically $1 \mathrm{mJy}$. So, from the optical spectrum and the radio flux, we can rule out both possibilities.

Along with the variation of coronal lines, a significant increase of [O III] emission lines is detected in the four TDE targets. There is no clear evidence that emission lines such as [O III] from normal AGN NLRs would show strong variability due to their large size. So persistent AGN activity is very unlikely to be the powering source of the variation of coronal lines and [O III] lines.

The disappearance of broad emission lines are also very different from the results of other Seyfert galaxy monitoring projects so far. In Seyfert galaxies, broad emission lines usually vary with a moderate amplitude in response to changes in the continuum. Only two cases were reported for broad emission lines temporally disappearing over a period of a few months accompanied by large continuum variations: NGC 4151 and NGC 5548 (Penston \& Perez, 1984; Iijima et al. 1992). In J0748+4712 and J0952+2143, which have several observations after the outburst, we only find broad lines that are either fading or have disappeared. Therefore, there is no evidence for the restarting of the central engine. Considering all these facts, we believe that the powering source is likely a strong outburst in an quiescent galaxy rather than the stochastic variability of an AGN.

\subsection{Could the Powering Source be A Supernova Explosion?}

The supernova $(\mathrm{SN})$ scenario is unable to explain the high luminosities of the coronal lines, the absence of other lowionization lines, and the continuum variability in some targets (W12). Also, the MMT follow-up observations provide additional constraints on the SN scenario. First, four TDE candidates show an increase of [O III] between the SDSS and MMT observations, and the [O III] lines are systematically broader than normal low-ionization lines such as $\mathrm{H} \alpha$ (Figure 8). These characteristics are different from other known SNe. Very few young SNe display variable narrow [O III] emission. Some $\mathrm{SNe}$ do show [O III] emission a few years to up $100 \mathrm{yr}$ after the explosion, but these lines are usually broad and accompany low-ionization lines of similar widths (Milisavljevic et al. 2012). These lines are formed in the SN shell interacting with the stellar winds. Second, based on the total energy in the high-ionization coronal lines, W12 estimated a total energy in soft X-ray of order $10^{50} \mathrm{erg}$. The emergence of [Fe VII] lines in these two objects (J1342+0530 \& J1350+2916), with only high-ionization coronal lines in previous SDSS spectra and the persistence of [Fe VII] in J0952+2143 suggest that a strong UV to X-ray ionizing continuum is still seen by the line-emitting gas. The brightening of the He II $\lambda 4686$ line in $\mathrm{J} 1342+0530$ indicates a further increase of extreme ultraviolet ionizing photons absorbed by line emission gas in this object, probably arising from a large covering factor of gas at the time lag. These variations of the [Fe VII] and He II $\lambda 4686$ lines reenforce the conclusion in W12 that a very energetic flare is required to power the coronal line emission.

As shown in the last section, the presence of an optically thick [O III] emission region requires large ionization parameters. In echo models, gas is photoionized by the continuum flare that took place 5-9 yr ago, so the size of the emission line region is $R_{[\mathrm{O}}$ II] $] 1-3 \mathrm{pc}$. The peak luminosity of ionizing continuum can then be estimated from the ionization parameter, the distance, and the density:

$$
\begin{aligned}
L_{\text {ion }}= & 4 \pi R_{[\mathrm{O} \text { III }]}^{2} n(\mathrm{H}) U(\mathrm{H}) c\langle h v\rangle>7.9 \times 10^{45} t_{4}^{-0.3} Z^{-1} \\
& \times R_{p c} n_{6}(\mathrm{H}) \mathrm{erg} \mathrm{s}^{-1}
\end{aligned}
$$

With a reasonable $n_{6}(\mathrm{H})=n(\mathrm{H}) / 10^{6} \mathrm{~cm}^{-3} \sim 1$, the luminosity would be $10^{45-46} \mathrm{erg} \mathrm{s}^{-1}$, which is much more luminous than any $\mathrm{SN}$ and is close to the Eddington limit for a $10^{7-8} M_{\odot}$ black hole. Finally, the lifetimes of broad lines are at most several years, which is much shorter than those of Type II SNe.

\section{CONCLUSIONS}

We have carried out follow-up MMT observations of seven extreme coronal line emitters that were studied by W12. Three objects turn out to be persistently strong coronal line emitters. Among them, two are likely star-forming and AGN composites or tidal disruptions of a giant star, and one is a narrowline Seyfert 1 galaxy. In the other four objects, all coronal lines with ionization lines higher than [Fe VII] disappear. Thus, they are tidal disruption candidates. [Fe VII] lines faded in J0952+2143 and appeared in the MMT spectra of two objects with higher ionization coronal lines only in the SDSS spectra. [O III] doublets appear brightened in all four objects. Thus, we are still witnessing echoes of the continuous decrease of the gas ionization in the emitting region. If this trend continues, the objects will appear in the AGN locus of the BPT diagram. Further monitoring of the emission lines is needed.

We also detected non-canonical [O III] ratios in two objects. The column density of $\mathrm{O}^{2+}$ must be large enough to make [O III $] 5007$ optically thick. For a reasonable metallicity, the $\mathrm{H}$ column density is large and the gas is probably optically thick to electron scattering. Lines will be broadened due to electron scattering, which can be tested with future high-quality data. The ionization parameter has to be very large to obtain this column density, and this also requires that the ionizing SED be relatively soft, equivalent to a $T<5 \times 10^{4} \mathrm{~K}$ blackbody, or oxygen would be too ionized. A gas temperature of the order of $10^{4} \mathrm{~K}$ will occur for such extreme conditions due to the nature of photoionization equilibrium, if the SED is this soft.

These observations can fit into a picture where giant molecular clouds are illuminated by continuum flares with an ionizing continuum luminosity of the order of $10^{45}-10^{46} \mathrm{erg} \mathrm{s}^{-1}$ within a few parsecs of the nucleus. The gas in the inner face of the cloud produces the coronal lines. As high-energy photons are absorbed by the gas, the ionizing continuum illuminating the outer part of the clouds softens and creates a thick [O III] emission region. The presence of giant molecular clouds in the central parsec and no persistent nuclear activity is striking, suggesting that perturbations in the gas are more fundamental for black hole fueling. Clearly, we still miss some critical evolution phases, which prevents us from probing a direct connection between these two subclasses of extreme coronal line emitters. Future follow-up of new discoveries of such objects from the spectroscopic surveys 
of LAMOST and BigBOSS can fill the gaps and yield a more complete picture of emission line evolution.

Broad emission lines previously seen in the SDSS spectra of J0748+4712 and J1342+0530 vanished in the MMT spectra, and there are only weak broad Balmer lines in J0952+2143. In J0952+2143, we observed continuous fading of the broad lines by a factor of 11 in the past $8 \mathrm{yr}$ as well as a decrease in the line width and velocity shift. The line profile variability perhaps reflects the deceleration of emission line gas in the gravitational field of the black hole due to a lack of further radiative acceleration. With improved subtraction of stellar light, the broad lines in these objects are found to all be double-peaked or blue-shouldered, suggesting that these are general properties probably arising from biconical outflows.

We thank Zheng Cai, Zhen-Ya Zheng, and Hui Dong for their help with the MMT observations and Stefanie Komossa for her very useful discussions. This work is supported by NSFC 11233002 and 10973013. This research uses data obtained through the Telescope Access Program (TAP), which is funded by the National Astronomical Observatories, Chinese Academy of Sciences, and the Special Fund for Astronomy from the Ministry of Finance. Observations reported here were obtained at the MMT Observatory, a joint facility of the University of Arizona and the Smithsonian Institution. G.J.F. acknowledges support from NSF (1108928 and 1109061) and STScI (HSTAR-12125.01, GO-12560, and HST-GO-12309).

Note added in proof. After this paper was submitted, an article on similar topics appeared on http://arxiv.org/abs/1304.6397.

\section{REFERENCES}

Asplund, M., Grevesse, N., Sauval, A. J., \& Scott, P. 2009, ARA\&A, 47, 481 Ayal, S., Livio, M., \& Piran, T. 2000, ApJ, 545, 772

Baldwin, J. A., Phillips, M. M., \& Terlevich, R. 1981, PASP, 93, 5

Becker, R. H., White, R. L., \& Helfand, D. J. 1995, ApJ, 450, 559
Bogdanović, T., Eracleous, M., Mahadevan, S., Sigurdsson, S., \& Laguna, P. 2004, ApJ, 610, 707

Brandt, W. N., Pounds, K. A., \& Fink, H. 1995, MNRAS, 273, L47

Bruzual, G., \& Charlot, S. 2003, MNRAS, 344, 1000

Cenko, S. B., Bloom, J. S., Kulkarni, S. R., et al. 2012, MNRAS, 420, 2684

Eracleous, M., Gezari, S., Grupe, D., Komossa, S., \& Saxton, R. 2012, European Physical Journal Web of Conferences, 39, 9001

Ferrarese, L., \& Merritt, D. 2000, ApJL, 539, L9

Gebhardt, K., Bender, R., Bower, G., et al. 2000, ApJL, 539, L13

Gezari, S., Chornock, R., Rest, A., et al. 2012, Natur, 485, 217

Gezari, S., Heckman, T., Cenko, S. B., et al. 2009, ApJ, 698, 1367

Grupe, D., Beuermann, K., Mannheim, K., et al. 1995, A\&A, 299, L5

Hills, J. G. 1975, Natur, 254, 295

Iijima, T., Rafanelli, P., \& Bianchini, A. 1992, A\&A, 265, L25

Kewley, L. J., Groves, B., Kauffmann, G., \& Heckman, T. 2006, MNRAS, 372,961

Komossa, S., \& Bade, N. 1999, A\&A, 343, 775

Komossa, S., Zhou, H., Rau, A., et al. 2009, ApJ, 701, 105

Komossa, S., Zhou, H., Wang, T., et al. 2008, ApJL, 678, L13

Kormendy, J., \& Richstone, D. 1995, ARA\&A, 33, 581

Laor, A. 2006, ApJ, 643, 112

Lodato, G., King, A. R., \& Pringle, J. E. 2009, MNRAS, 392, 332

Lu, H., Zhou, H., Wang, J., et al. 2006, AJ, 131, 790

Magorrian, J., Tremaine, S., Richstone, D., et al. 1998, AJ, 115, 2285

Milisavljevic, D., Fesen, R. A., Chevalier, R. A., et al. 2012, ApJ, 751, 25

Osterbrock, D. E., \& Ferland, G. J. (ed.) 2006, Astrophysics of Gaseous Nebulae and Active Galactic Nuclei (2nd ed.; Sausalito, CA: University Science Books)

Penston, M. V., \& Perez, E. 1984, MNRAS, 211, 33P

Penston, M. V., Fosbury, R. A. E., Boksenberg, A., Ward, M. J., \& Wilson, A. S. 1984, MNRAS, 208, 347

Peterson, B. M., \& Ferland, G. J. 1986, Natur, 324, 345

Rees, M. J. 1988, Natur, 333, 523

Richards, G. T., Kruczek, N. E., Gallagher, S. C., et al. 2011, AJ, 141, 167

Storey, P. J., \& Zeippen, C. J. 2000, MNRAS, 312, 813

Strubbe, L. E., \& Quataert, E. 2009, MNRAS, 400, 2070

Ulmer, A. 1999, ApJ, 514, 180

van Velzen, S., Farrar, G. R., Gezari, S., et al. 2011, ApJ, 741, 73

Veilleux, S. 1988, AJ, 95, 1695

Wang, H., Wang, T., Zhou, H., et al. 2011, ApJ, 738, 85

Wang, T.-G., Zhou, H.-Y., Komossa, S., et al. 2012, ApJ, 749, 115

Wang, T.-G., Zhou, H.-Y., Wang, L.-F., Lu, H.-L., \& Xu, D. 2011, ApJ, 740,85 